\title{
Plumbagin Protects Mice from Lethal Sepsis by Modulating Immunometabolism Upstream of PKM2
}

\author{
Zhaoxia Zhang, ${ }^{1}$ Wenjun Deng, ${ }^{2}$ Rui Kang, ${ }^{3}$ Min Xie, ${ }^{2}$ Timothy Billiar, ${ }^{3}$ Haichao Wang, ${ }^{4}$ Lizhi Cao, ${ }^{2}$ and \\ Daolin Tang $g^{3,5}$
}

${ }^{1}$ Department of Pediatrics, The Second Affiliated Hospital of Jinan University, Shenzhen People's Hospital, Shenzhen, Guangdong, People's Republic of China; ${ }^{2}$ Department of Pediatrics, Xiangya Hospital, Central South University, Changsha, Hunan, People's Republic of China; ${ }^{3}$ Department of Surgery, University of Pittsburgh, Pittsburgh, Pennsylvania, United States of America; ${ }^{4}$ Laboratory of Emergency Medicine, The Feinstein Institute for Medical Research, Manhasset, New York, United States of America; and ${ }^{5}$ Center for DAMP Biology, The Third Affiliated Hospital of Guangzhou Medical University, Guangzhou, Guangdong, People's Republic of China

\begin{abstract}
Sepsis is characterized by dysregulated systemic inflammation with release of early (for example, interleukin (IL)-1 $\beta$ ) and late (for example, HMGB1) proinflammatory mediators from macrophages. Plumbagin, a medicinal plant-derived naphthoquinone, has been reported to exhibit antiinflammatory activity, but the underling mechanisms remain unclear. Here, we have demonstrated that plumbagin inhibits the inflammatory response through interfering with the immunometabolism pathway in activated macrophages. Remarkably, plumbagin inhibited lipopolysaccharide (LPS)-induced aerobic glycolysis by downregulating the expression of pyruvate kinase M2 (PKM2), a protein kinase responsible for the final and rate-limiting reaction step of the glycolytic pathway. Moreover, the NADPH oxidase 4 (NOX4)-mediated oxidative stress was required for LPS-induced PKM2 expression, because pharmacologic or genetic inhibition of NOX4 by plumbagin or RNA interference limited LPS-induced PKM2 expression, lactate production and subsequent proinflammatory cytokine (IL-1 $\beta$ and HMGB1) release in macrophages. Finally, plumbagin protected mice from lethal endotoxemia and polymicrobial sepsis induced by cecal ligation and puncture. These findings identify a new approach for inhibiting the NOX4/PKM2-dependent immunometabolism pathway in the treatment of sepsis and inflammatory diseases.
\end{abstract}

Online address: http://www.molmed.org

doi: $10.2119 / \mathrm{molmed} .2015 .00250$

\section{INTRODUCTION}

Bacterial infections leading to sepsis and septic shock remain a major reason for admission to intensive care units (1). Lipopolysaccharide (LPS), the major component of the outer membrane of Gram-negative bacteria, is a critical activator of macrophage release of proinflammatory mediators such as interleukin (IL)-1 $\beta$ and high mobility group box 1 (HMGB1). In contrast to early proinflammatory cytokines (for example, IL-1 $\beta$ ) (2), HMGB1 is released by macrophages in a delayed manner, and thus functions as a late mediator of lethal sepsis (3). In addition to its direct proinflammatory activity, extracellular HMGB1 can also amplify the inflammatory response evoked by multiple pathogenassociated molecular patterns (PAMPs)
Submitted December 6, 2015; Accepted for publication February 16, 2016; Published Online (www.molmed.org) March 9, 2016.

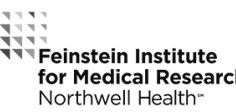

Feinstein Institute
for Medical Research Northwell Health

\begin{abstract}
Address correspondence to Lizhi Cao, Department of Pediatrics, Xiangya Hospital, Central South University, 110 Xiangya Road Changsha, Hunan 410008, People's Republic of China. Phone: +86-737-84327208; Fax:+86-737-84327332; E-mail: lizhicao@gmail.com or Daolin Tang, Department of Surgery, University of Pittsburgh, G.27C, Hillman Cancer Center, 5117 Centre Ave, Pittsburgh PA, 15213, USA. Phone: 412-623-1211; Fax: 412-623-1212; E-mail: tangd2@upmc.edu
\end{abstract}

and other damage-associated molecular patterns (DAMPs) (4). These immunostimulatory properties and kinetics of its delayed release make HMGB1 a promising therapeutic target for sepsis $(5,6)$.

To better understand the complicated pathogenesis of sepsis, it is important to define intricate molecular mechanisms and signaling pathways underlying the regulation of HMGB1 release and proinflammatory activities. As tightly regulated processes, the innate immune response and metabolism are highly integrated (7-9). When oxygen supply is limited, aerobic glycolysis enables the conversion of glucose to pyruvate with the involvement of several enzymes including pyruvate kinase M2 (PKM2), a protein kinase for the final and rate-limiting reaction step of the glycolytic pathway. We recently demonstrated that PKM2 is markedly upregulated in activated macrophages and that PKM2-mediated aerobic 
glycolysis contributes the pathogenesis of sepsis through the regulated release of HMGB1 (10). However, the mechanism underlying the dramatic upregulation of PKM2 expression remains undefined.

Plumbagin (5-hydroxy-2-methyl-1, 4-naphthoquinone) is a quinone isolated from the roots of Plumbago zeylanica. At higher doses, plumbagin ( $>30 \mu \mathrm{mol} / \mathrm{L}$ ) induces cell cycle arrest and apoptosis in some cancer cells $(11,12)$. In contrast, low-dose plumbagin $(<5 \mu \mathrm{mol} / \mathrm{L})$ cannot induce cell death, but exhibits antiinflammatory activity in macrophages and $\mathrm{T}$ cells (13-15). In this study, we provide new evidence regarding the antiinflammatory function of plumbagin and its mechanism of action in macrophages. We demonstrate that plumbagin attenuates LPS-induced HMGB1 release in macrophages by inhibiting NADPH oxidase 4 (NOX4)-mediated PKM2 expression. These findings significantly enhance the understanding of the functional consequences of immunometabolism in sepsis and identify a potentially new targeted therapy to reduce the morbidity of sepsis.

\section{MATERIALS AND METHODS}

\section{Reagents}

The antibodies to PKM2 (\#4053) and actin (\#3700) were obtained from Cell Signaling Technology. The antibody to NOX4 (\#UOTR1B493) was obtained from Abcam. LPS (Escherichia coli LPS 0111:B4; \#L4391), 2DG (\#D8375) and plumbagin (\#P7262) were obtained from Sigma. GKT137831 (\#S7171) was obtained from Selleck Chemicals.

\section{Cell Culture}

Mouse peritoneal macrophages (PMs) and bone marrow-derived macrophages (BMDMs) were isolated from Balb/C mice as previously described $(16,17)$. For isolation of PMs, normal mice were injected intraperitoneally (i.p.) with $1.5 \mathrm{~mL}$ of 3\% Brewer's thioglycollate broth for 3 d. Primary PMs were collected from euthanized normal or sepsis animals by peritoneal lavage using $10 \mathrm{~mL}$ of ice cold RPMI supplemented with $2 \%$ fetal bovine serum (FBS), 1 unit/mL heparin and penicillin/streptomycin. Cells were washed using lavage media without heparin and plated in macrophage culture media of Dulbecco's modified Eagle's medium (DMEM) supplemented with 10\% heat-inactivated FBS and penicillin/streptomycin and incubated at $37^{\circ} \mathrm{C}$ at $5 \% \mathrm{CO}_{2}$ for $2 \mathrm{~h}$. Cultures were washed three times with phosphate buffered saline (PBS) to remove non-adherent cells and retain adherent cells (PMs) in the culture media.

For isolation of BMDMs, bone marrow cells were isolated from femurs and tibias. Cells were then incubated for $7 \mathrm{~d}$, the period required for bone marrow cells to differentiate into macrophages, in DMEM containing 10\% FBS and supplemented with 30\% L929 medium. The medium was replenished on the fourth day. Cultures were washed three times with PBS to remove non-adherent cells and retain adherent cells (BMDMs) in the culture media.

\section{Oxidative Phosphorylation and Glycolysis Assay}

Cellular OXPHOS and glycolysis were monitored using the Seahorse Bioscience Extracellular Flux Analyzer (XF24) by measuring the oxygen consumption rate (OCR) (indicative of respiration) and the extracellular acidification rate (ECAR) (indicative of glycolysis) $(18,19)$. Briefly, 30,000 to 50,000 cells were seeded in 24-well plates designed for XF24 in $150 \mu \mathrm{L}$ of appropriate growth media and incubated overnight. Prior to measurements, cells were washed with unbuffered media once, then immersed in $675 \mu \mathrm{L}$ unbuffered media and incubated in the absence of $\mathrm{CO}_{2}$ for $1 \mathrm{~h}$. OCR and ECAR were then measured in a typical 8-min cycle of mix (2 to $4 \mathrm{~min}$ ), dwell (2 $\mathrm{min}$ ) and measurement ( 2 to $4 \mathrm{~min}$ ) by using Mito Stress Test Kit (Part \# 103015-100; Oligomycin, $100 \mu \mathrm{M}$; carbonyl cyanide p-trifluoromethoxyphenylhydrazone [FCCP], $100 \mu \mathrm{mol} / \mathrm{L}$; Rotenone/ antimycin A; $50 \mu \mathrm{mol} / \mathrm{L}$ ) and Glycolysis Stress Test Kit (Part \# 103020-100;
Glucose, $10 \mathrm{mM}$; Oligomycin, $1 \mu \mathrm{M}$; 2-DG, $50 \mu \mathrm{mol} / \mathrm{L}$ ) from Seahorse Bioscience.

\section{Cytokine Measurements}

Commercially available enzyme-linked immunosorbent assay (ELISA) kits were used to measure the concentrations of HMGB1 and IL-1 $\beta$ in serum or the culture medium according to the manufacturer's instructions.

\section{RNAi and Gene Transfection}

Transfection with NOX4-shRNA-1 (5'-CCGGG CATCA AATAA CCACC TGTAT CTCGA GATAC AGGTG GTTAT TTGAT GCTTT TTG-3'), NOX4shRNA-2 (5'-CCGGG CCAGT ATATT ATTCT CCATT CTCGA GAATG GAGAA TAATA TACTG GCTTT TTG$3^{\prime}$ ) and control shRNA (SHC001) from Sigma were performed using MISSION shRNA Lentiviral Transduction. Transfection with mouse pCMV6-NOX4 cDNA from OriGene Technologies (\#MC219182) was performed using the Neon ${ }^{\mathrm{TM}}$ Transfection System according to the manufacturer's instructions.

\section{Western Blot Analysis}

Proteins in cell lysates were first resolved by SDS-polyacrylamide gel electrophoresis and then transferred to nitrocellulose membrane and subsequently incubated with the primary antibody as previously described (20). After incubation with peroxidase-conjugated secondary antibodies, the signals were visualized using enhanced chemiluminescence according to the manufacturer's instruction. Native gel electrophoresis assays were performed to evaluate the formation of dimeric PKM2.

\section{Quantitative Real-Time Polymerase Chain Reaction}

Total RNA was extracted using TRI reagent according to the manufacturer's instructions. First-strand cDNA was synthesized from $1 \mu \mathrm{g}$ of RNA using the iScript cDNA Synthesis kit. cDNA from various cell samples was amplified by real-time quantitative PCR 
with specific primers (PKM2: 5'-TCGCA TGCAG CACCT GATT-3' and 5'-CCTCG AATAG CTGCA AGTGG TA-3') and the data was normalized to $18 \mathrm{~S}$ ribosomal RNA (5'-CTTAG AGGGA CAAGT GGCG-3' and 5'-ACGCT GAGCC AGTCA GTGTA-3').

\section{Lactate and Phosphoenolpyruvate Assay}

Lactate in the culture medium or serum was measured with colorimetric L-Lactate Assay Kit according to the manufacturer's instructions. Phosphoenolpyruvate (PEP) production was measured using a PEP fluorometric assay kit according to the manufacturer's instructions.

\section{NOX Activity Assay}

NOX activity was measured by lucigenin chemiluminescence assay as previously described (21). Briefly, cultured cells were homogenized in lysis buffer $\left(20 \mathrm{mmol} / \mathrm{L} \mathrm{KH}_{2} \mathrm{PO}_{4}\right.$, $\mathrm{pH}$ 7.0, $1 \mathrm{mmol} / \mathrm{L}$ ethylene glycol tetraacetic acid (EGTA), $1 \mathrm{mmol} / \mathrm{L}$ phenylmethylsulfonyl fluoride, $10 \mu \mathrm{g} / \mathrm{mL}$ aprotinin and $0.5 \mu \mathrm{g} / \mathrm{mL}$ leupeptin). Aliquots $(100 \mu \mathrm{L})$ of homogenates were then added to $900 \mu \mathrm{L}$ of $50 \mathrm{mmol} / \mathrm{L}$ phosphate buffer, $\mathrm{pH} 7.0$, containing $1 \mathrm{mmol} / \mathrm{L}$ EGTA, $150 \mathrm{mmol} / \mathrm{L}$ sucrose, $5 \mu \mathrm{mol} / \mathrm{L}$ lucigenin and $100 \mu \mathrm{mol} / \mathrm{L}$ NADPH. The photon emission for NOX-mediated superoxide anion production was measured using a luminometer.

\section{Animal Model of Endotoxemia and Sepsis}

This study was approved and performed in accordance with guidelines for the care and use of laboratory animals at Central South University, Changsha, China. Endotoxemia was induced in Balb/C mice (male, 7 to 8 wks old, 20 to $25 \mathrm{~g}$ weight) by i.p. injection of bacterial endotoxin (LPS, $5 \mathrm{mg} / \mathrm{kg}$ ) as previously described $(3,22)$. Sepsis was induced in male Balb/C mice (male, 7 to 8 wks old, 20 to $25 \mathrm{~g}$ weight) by cecal ligation and puncture (CLP) as previously described
$(3,22-24)$. Briefly, anesthesia was induced with $5 \%$ halothane and maintained with $2 \%$ halothane. A small midline abdominal incision was made and the cecum was exteriorized and ligated with 4-0 silk immediately distal to the ileocecal valve without causing intestinal obstruction. The cecum was then punctured once with an 18 gauge needle. The abdomen was closed in two layers, and the mice were injected subcutaneously with $1 \mathrm{~mL}$ of $0.9 \%$ $\mathrm{NaCl}$ including analgesia $(0.05 \mathrm{mg} / \mathrm{kg}$ buprenorphine). After CLP, mice did not receive antibiotics.

Plumbagin was dissolved in vehicle (10\% DMSO, 20\% cremophor:ethanol [3:1] and 70\% PBS) and administered i.p. to mice at the indicated time points. Blood was collected at indicated time points, allowed to clot for $2 \mathrm{~h}$ at room temperature and then centrifuged for $15 \mathrm{~min}$ at $1,500 \times \mathrm{g}$. Serum samples were stored at $-20^{\circ} \mathrm{C}$ before analysis. Mortality was recorded for up to 2 to 3 wks after the onset of lethal endotoxemia or sepsis to ensure that no additional late deaths occurred.

\section{Statistical Analysis}

Data are expressed as means \pm SEM of three independent experiments. Significance of differences between groups was determined using ANOVA LSD test. The Kaplan-Meier method was used to compare differences in mortality rates between groups. Analysis was performed using SigmaPlot 11.0. A $p$ value $<0.05$ was considered statistically significant.

All supplementary materials are available online at www.molmed.org.

\section{RESULTS}

\section{Plumbagin Inhibits Aerobic Glycolysis in Activated Macrophages}

To investigate whether plumbagin affects aerobic glycolysis, we evaluated oxidative phosphorylation (as measured by OCR) and glycolysis (as measured by ECAR) in activated BMDMs following LPS (100 ng/mL) treatment. At low doses (1 to $3 \mu \mathrm{mol} / \mathrm{L})$, plumbagin did not affect cell viability (Figure 1A), but significantly inhibited the LPS-induced switch from oxidative phosphorylation to glycolysis in a dose-dependent manner (Figures 1B, C). Two-deoxy-D-glucose (2-DG) is a widely-used competitive inhibitor of the first hexokinase (HK) of the glycolytic pathway (25). Consistent with previous studies $(10,26,27), 2-D G$ also inhibited the LPS-induced switch from oxidative phosphorylation to glycolysis (Figures 1B, C). We further analyzed the levels of glycolytic metabolites (for example, phosphoenolpyruvate [PEP] and lactate) in macrophages. As expected, both plumbagin and 2-DG inhibited the increase of PEP and lactate levels in LPS-stimulated BMDMs and PMs (Figure 1D). These findings indicate that plumbagin inhibits LPS-induced aerobic glycolysis in activated macrophages.

\section{Plumbagin Inhibits PKM2 Expression in Activated Macrophages}

Our previous study demonstrated that the upregulation of PKM2 is required for LPS-induced glycolysis in macrophages (10). To investigate whether plumbagin inhibits aerobic glycolysis through regulating PKM2 expression, we analyzed the mRNA and protein levels of PKM2 in activated BMDMs and PMs. Remarkably, plumbagin dose-dependently suppressed LPS-induced PKM2 expression at both mRNA (Figure 2A) and protein levels (Figure 2B). In contrast, plumbagin did not affect LPS-induced HK-1 mRNA expression (Figure 2C). Consistent with previous studies $(10,26)$, the protein expression of PKM1 did not change in macrophages following LPS treatment (Figure 2B). In addition to monomeric PKM2, dimeric PKM2 is a transcription cofactor of hypoxia-inducible factor 1- $\alpha$ (HIF1 $\alpha)$, which contributes to aerobic glycolysis. We previously demonstrated that knockdown of PKM2 inhibited LPS-induced HIF1 $\alpha$ activity in macrophages (10). As expected, native gel electrophoresis assay showed that plumbagin inhibited 
A

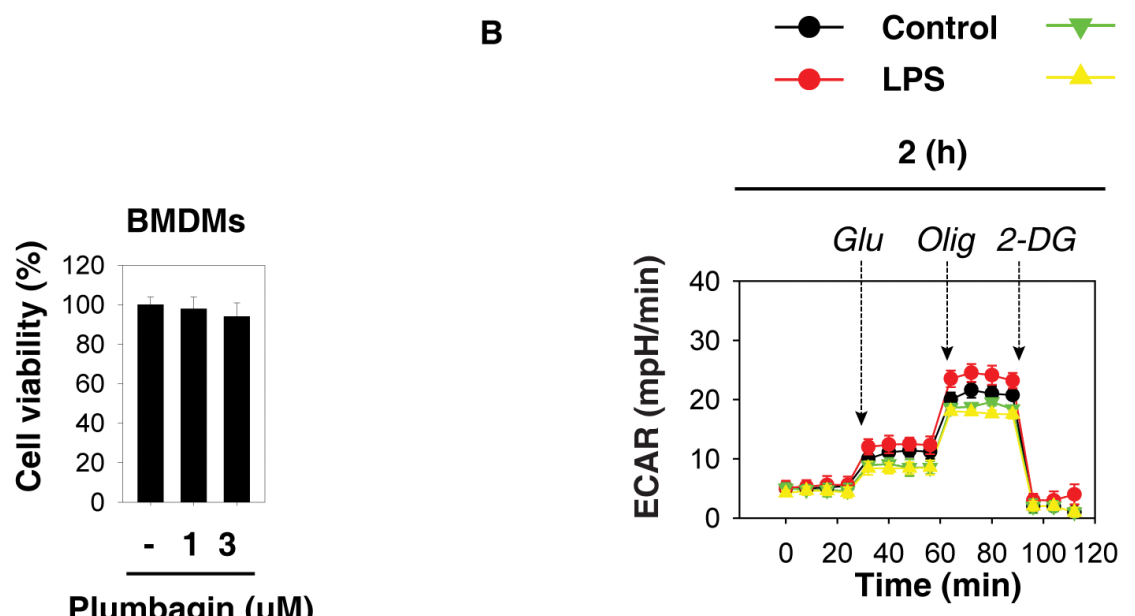

Plumbagin $(\mu \mathrm{M})$

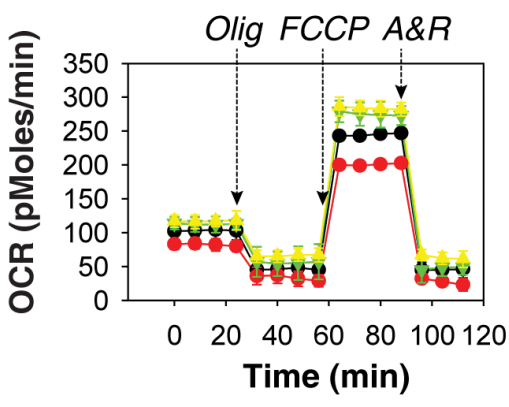

LPS+Plumbagin $(3 \mu \mathrm{M})$

LPS+2DG (2 mM)
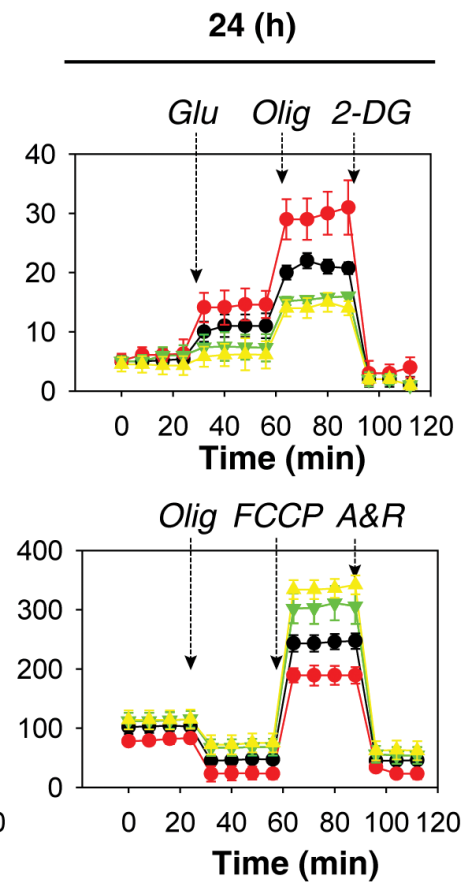

C

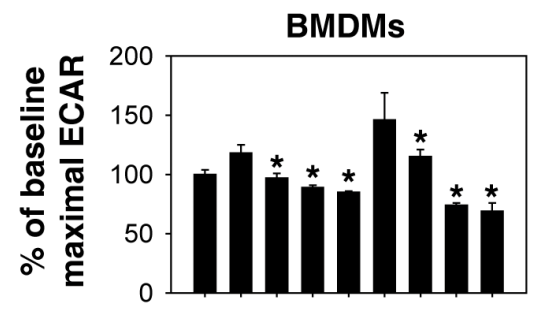

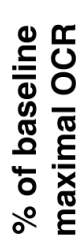

Plumbagin $(\mu \mathrm{M})$

2DG (mM)

LPS
D
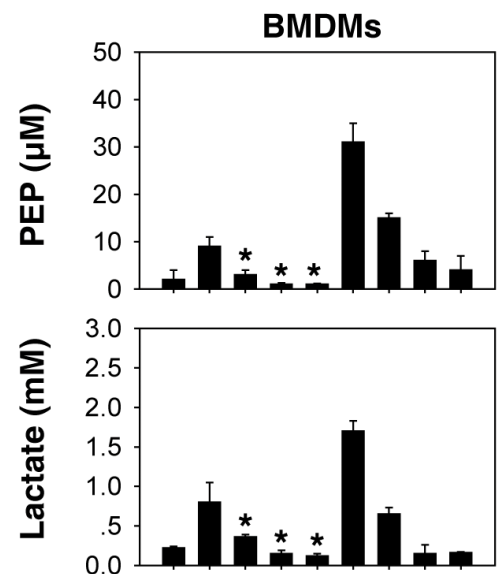

Plumbagin $(\mu \mathrm{M})-{ }^{-1} 3--13$

2DG (mM) - - - 2 - - 2

LPS
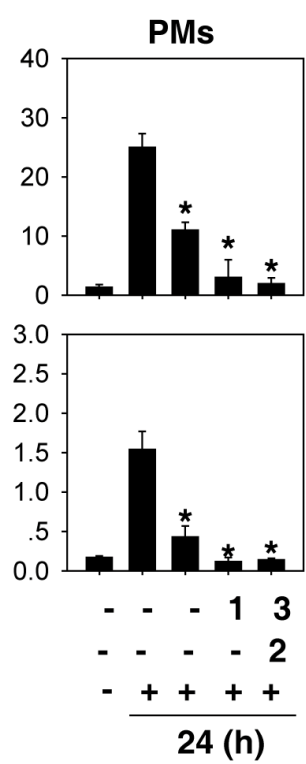

Figure 1. Plumbagin inhibits aerobic glycolysis in activated macrophages. (A) Bone marrow-derived macrophages (BMDMs) were treated with plumbagin ( 1 and $3 \mu \mathrm{mol} / \mathrm{L}$ ) for $24 \mathrm{~h}$, and cell viability was analyzed. (B-C) BMDMs and peritoneal macrophages (PMs) were pretreated with plumbagin and 2DG for $1 \mathrm{~h}$ and then stimulated with lipopolysaccharide (LPS) (100 ng/mL) for 2 or $24 \mathrm{~h}$. After the drug treatment ended, the cells were changed to fresh culture medium and then the oxygen consumption rates (OCR, indicative of oxidative phosphorylation) and extracellular acidification rates (ECAR, indicative of glycolysis) were monitored using the Mito Stress Test

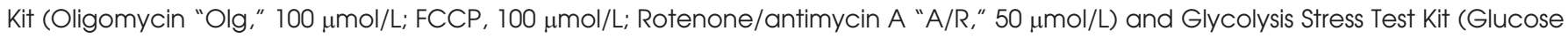
"Glu," $10 \mathrm{mM}$; Oligomycin "Olg," 1 m mol/L; 2-DG, $50 \mu \mathrm{mol} / \mathrm{L}$ ) by Seahorse Bioscience Extracellular Flux Analyzer. (D) In parallel, phosphoenolpyruvate (PEP) and lactate levels were assayed using commercial kits $\left(n=3,{ }^{*}, p<0.05\right.$ versus LPS group). 
A

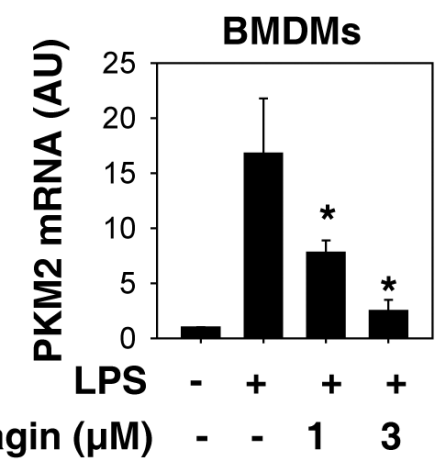

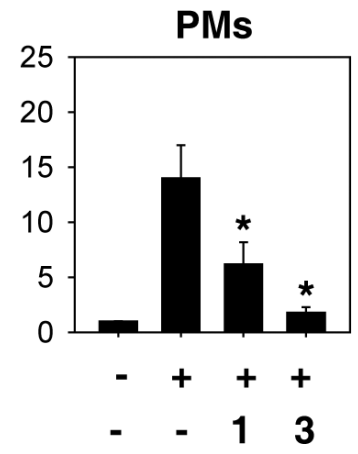

C

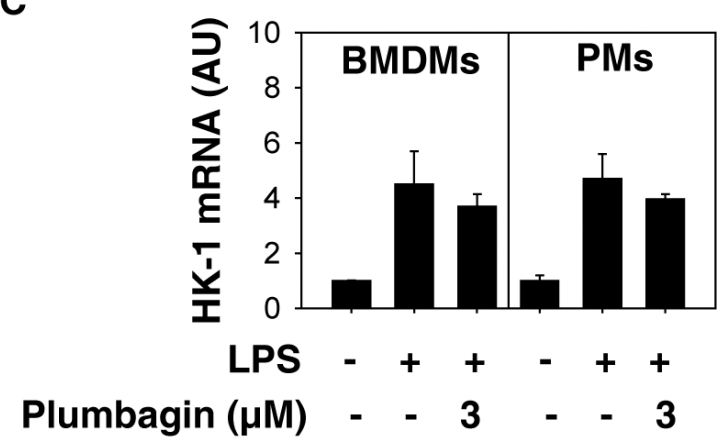

B

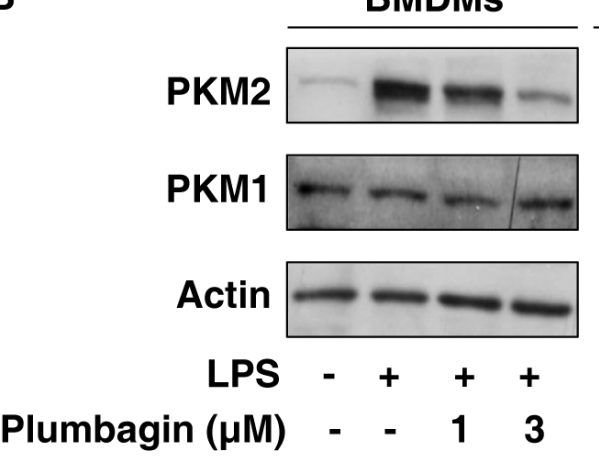

PMs

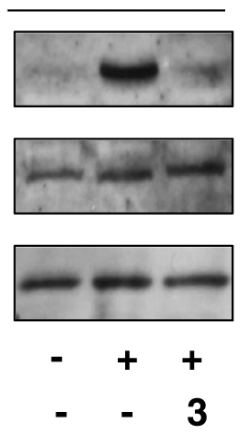

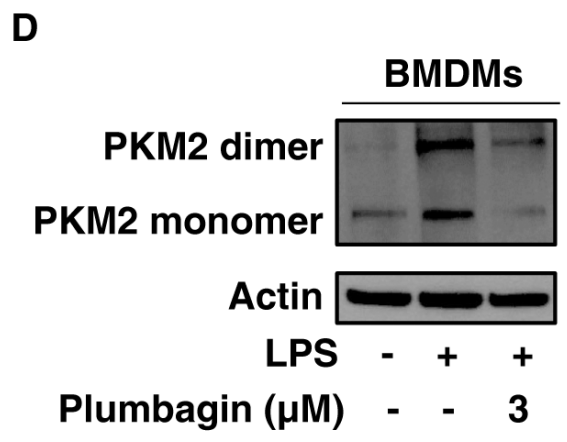

Figure 2. Plumbagin inhibits PKM2 expression in activated macrophages. (A-C) Indicated macrophages were treated with lipopolysaccharide (LPS) (100 ng/mL) in the absence or presence of plumbagin for $24 \mathrm{~h}$. The mRNA or protein levels of PKM2 (A-B) or HK-1 (C) were assayed using Q-PCR and western blot, respectively $\left(n=3,{ }^{*}, p<0.05\right.$ versus LPS group). (D) Native gel electrophoresis was performed using whole-cell extracts from BMDMs after treatment with LPS (100 ng/mL) and/or plumbagin (3 $\mu \mathrm{mol} / \mathrm{L})$ for $24 \mathrm{~h}$.

LPS-induced formation of dimeric PKM2 (Figure 2D). These findings suggest that plumbagin inhibits aerobic glycolysis partly through downregulating PKM2 expression in activated macrophages.

\section{NOX4 Is Required for PKM2 Expression in Glycolysis}

NOX4 is a constitutively active multi-subunit enzyme that generates superoxide from molecular oxygen using NADPH as the electron donor (28). It acts as an oxygen sensor and catalyzes the reduction of molecular oxygen to various reactive oxygen species (ROS) in macrophages (29). Given that plumbagin is a potent inhibitor of NOX4 activity (30-32), we next investigated whether NOX4-mediated oxidative stress is required for PKM2 expression in activated macrophages. Knockdown of NOX4 by two different specific NOX4-shRNAs in BMDMs and
PMs (Figure 3A) significantly inhibited LPS-induced ROS generation, PKM2 mRNA expression, and lactate production (Figure 3B). Moreover, GKT137831, a novel NOX1/NOX4 inhibitor $(33,34)$, also suppressed LPS-induced PKM2 mRNA expression and lactate production (Figure 3C) in BMDMs. Thus, the activation of NOX4 is required for LPS-induced PKM2 mRNA expression and subsequent aerobic glycolysis in macrophages.

NOX4 Is Required for IL- $1 \beta$ and HMGB 1 Release in Activated Macrophages

We previously demonstrated that pharmacologic and genetic inhibition of PKM2 attenuated LPS-induced IL-1 $\beta$ and HMGB1 release in macrophages (10). We therefore determined whether inhibition of NOX4 expression and activity also affects the release of these cytokines in macrophages. Suppression of NOX4 expression by specific shRNA significantly reduced LPS-induced IL-1 $\beta$ and HMGB1 release at early and late stages, respectively (Figure 4A). Similarly, NOX4 inhibitors (plumbagin and GKT137831) also limited LPS-induced IL-1 $\beta$ and HMGB1 release in macrophages (Figure 4B).

We next investigated whether overexpression of NOX4 expression affects the expression of PKM2 and release of cytokines in macrophages. Indeed, overexpression of NOX4 by gene transfection (Figure 4C) increased LPSinduced PKM2 mRNA expression and cytokine (IL-1 $\beta$ and HMGB1) release in BMDMs at early or late stages. This process in NOX4-overexpressed cells can be blocked by plumbagin. Collectively, these findings support a novel role of NOX4 in the regulation of proinflammatory 
A

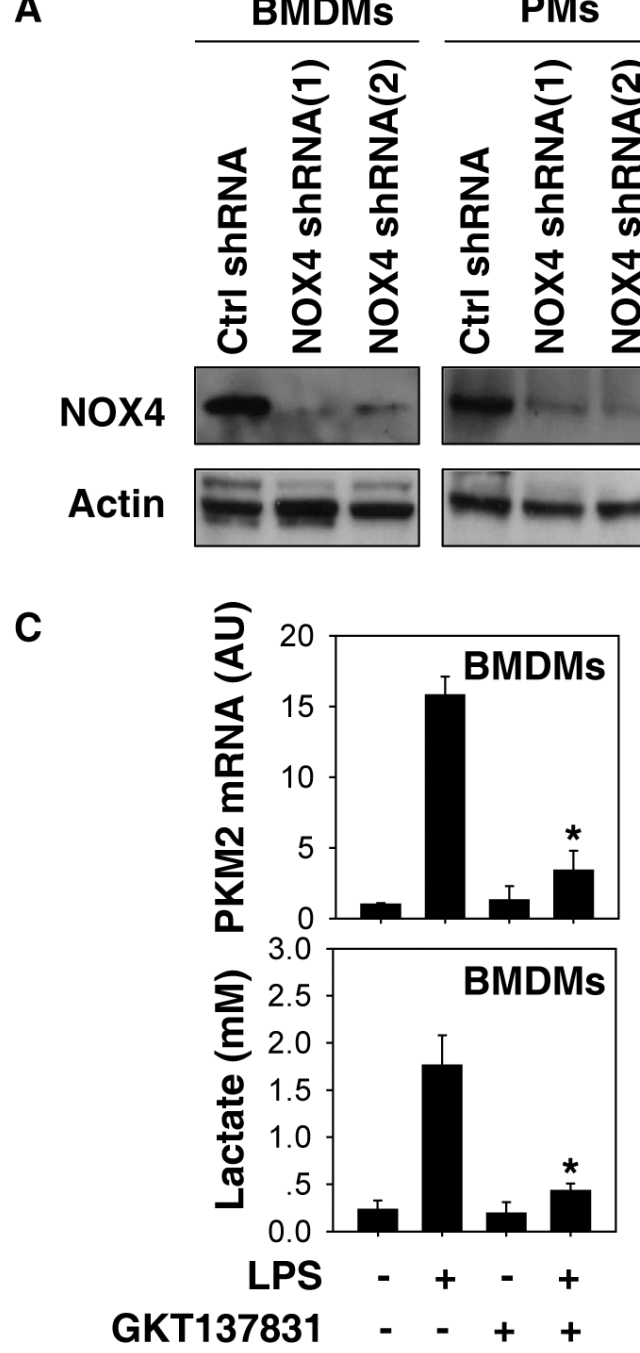

B

\section{$\square$ Ctrl shRNA \\ $\square$ NOX4 ShRNA(1) \\ NOX4 ShRNA(2)}
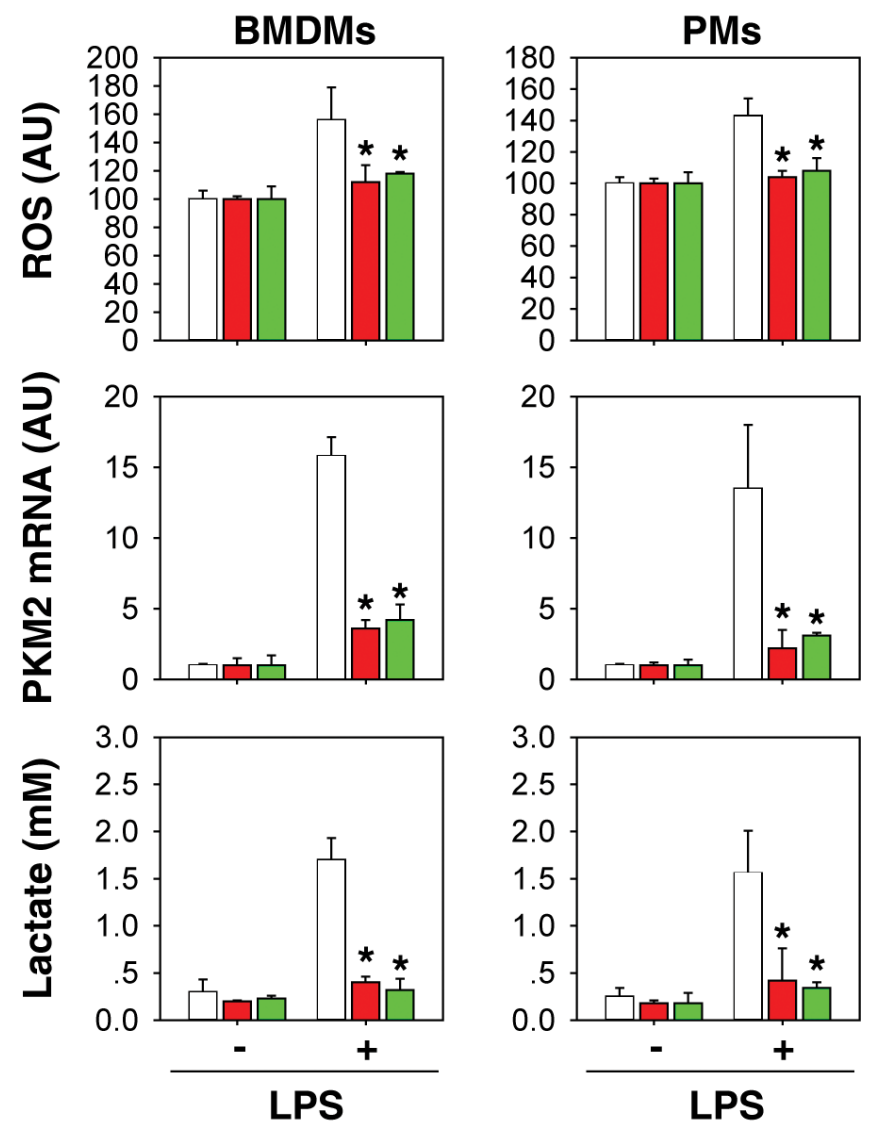

Figure 3. NOX4 is required for PKM2 expression in glycolysis. (A) Western blot analysis of NOX4 expression in control and PKM2 shRNA-treated bone marrow-derived macrophages (BMDMs) and peritoneal macrophages (PMs). (B) Indicated macrophages were treated with lipopolysaccharide (LPS) $(100 \mathrm{ng} / \mathrm{mL})$ for $24 \mathrm{~h}$. Relative reactive oxygen species (ROS), PKM2 mRNA, and lactate were assayed $\left(n=3,{ }^{*}, p<0.05\right.$ versus control shRNA group). (C) Indicated macrophages were treated with LPS (100 ng/mL) in the absence or presence of GKT137831 ( $1 \mu \mathrm{mol} / \mathrm{L})$ for $24 \mathrm{~h}$. PKM2 mRNA and lactate levels were assayed $\left(n=3,{ }^{*}, p<0.05\right.$ versus LPS group).

cytokine release by modulating PKM2 expression.

\section{Plumbagin Protects Mice from Endotoxic Shock and Sepsis}

Given that plumbagin has been used in clinical trials (35), we determined whether plumbagin protects mice against lethal endotoxemia by inhibiting cytokine release. Repetitive administration of plumbagin at $-0.5,+12$ and $+24 \mathrm{~h}$ following the onset of endotoxemia ( $5 \mathrm{mg} / \mathrm{kg}$ LPS, i.p.) conferred significant protection against lethal endotoxemia (Figure 5A). Serum levels of lactate (Figure 5B), IL-1 $\beta$ (Figure 5C), TNF (Figure 5D), HMGB1 (Figure 5E) and mRNA expression of PKM2 (Figure 5F), and NOX activity (Figure 5G) in PMs isolated from endotoxemic mice were lower after administration of plumbagin. As expected, ECAR showed that PMs isolated from the plumbagin treatment group had lower levels of glycolysis (Figure 5H).

We then used the CLP sepsis model to evaluate the ability of plumbagin to protect mice from polymicrobial intra-abdominal sepsis. At $+24,+48$ and $+72 \mathrm{~h}$ following the onset of sepsis, animals were given plumbagin or vehicle (i.p.). Plumbagin rescued mice from CLP-induced lethal sepsis, even if given after the onset of sepsis (Figure 6A). The serum levels of lactate (Figure 6B) and HMGB1 (Figure 6C), as well as PKM2 mRNA expression (Figure 6D) and NOX activity (Figure 6E) in PMs, were significantly lower in the group receiving plumbagin 
A
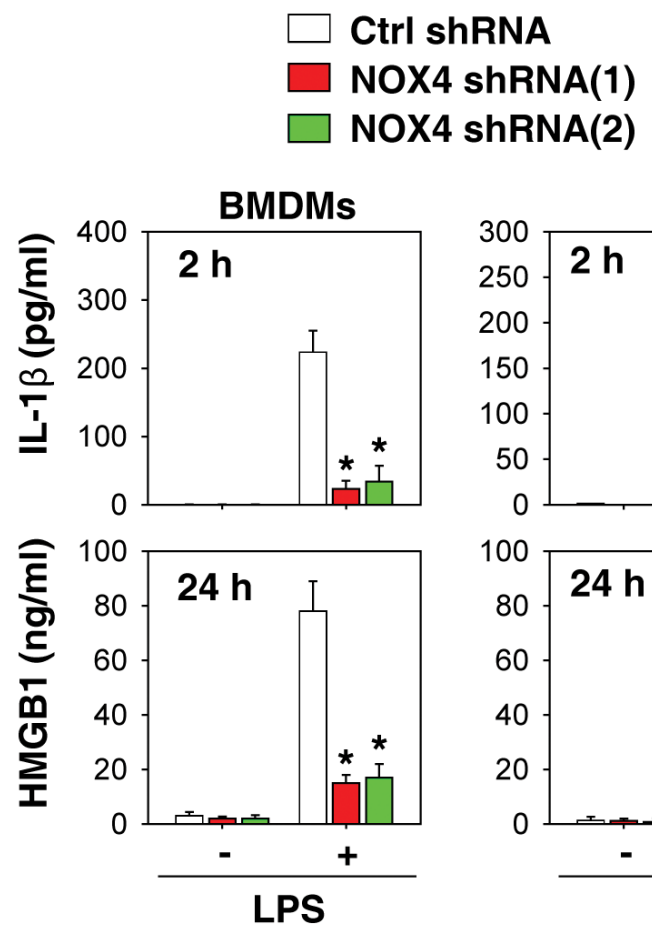

B
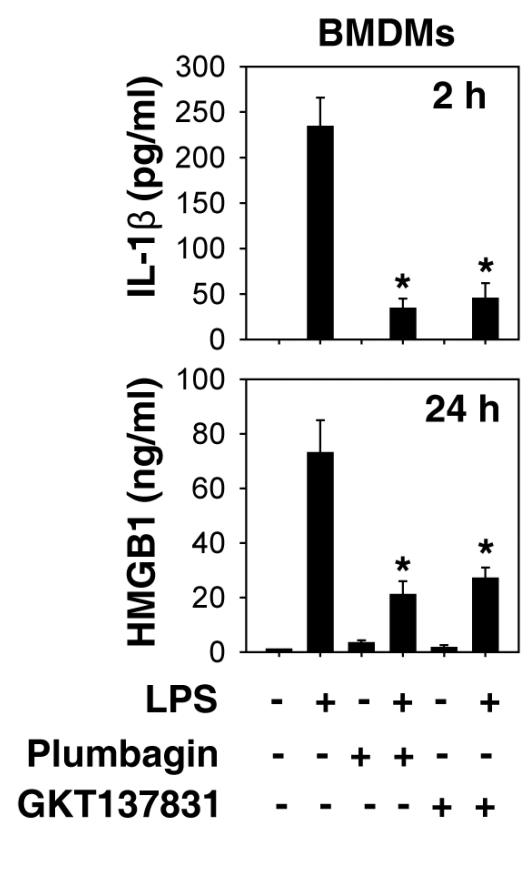

LPS
C

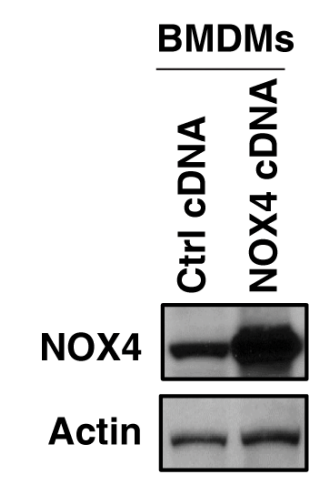

D
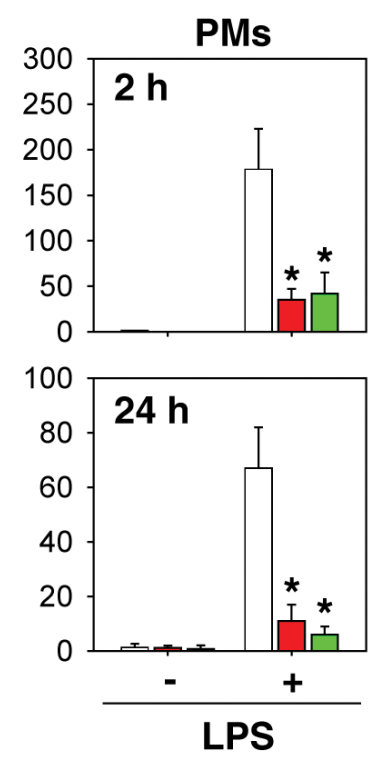
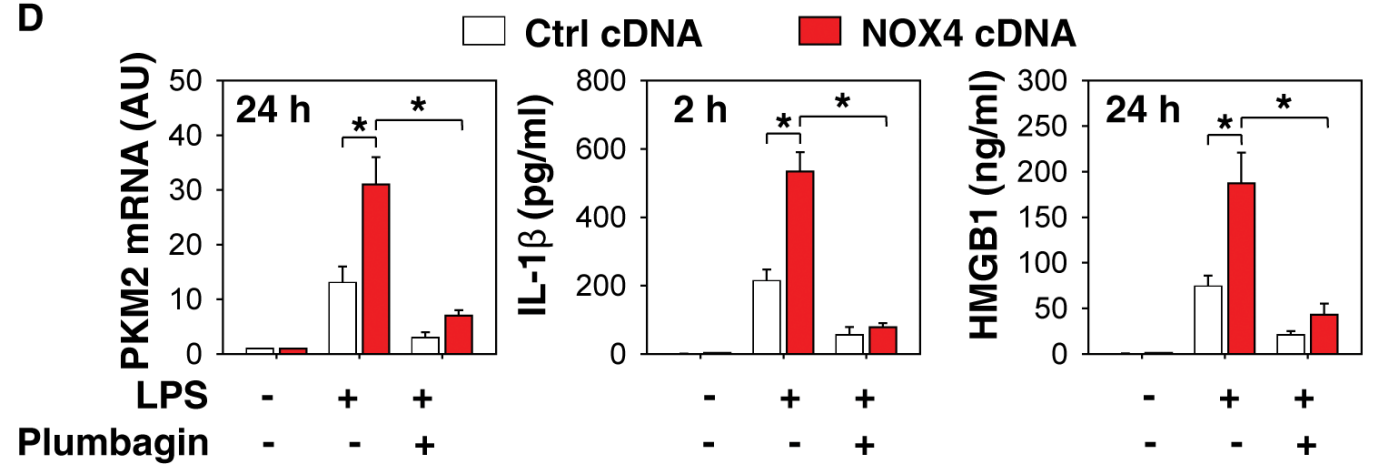

Figure 4. NOX4 is required for IL-1 $\beta$ and HMGB1 release in activated macrophages. (A) Indicated macrophages were treated with lipopolysaccharide (LPS) (100 ng/mL) and IL- $1 \beta$ levels at $2 \mathrm{~h}$ and high mobility group box 1 (HMGB1) at $24 \mathrm{~h}$ in supernatants were assayed using ELISA ( $n=3,{ }^{*}, p<0.05$ versus control shRNA group). (B) Bone marrow-derived macrophages (BMDMs) were treated with LPS (100 ng/mL) in the absence or presence of plumbagin $(3 \mu \mathrm{mol} / \mathrm{L})$ and $\mathrm{GKT} 137831(1 \mu \mathrm{mol} / \mathrm{L})$ and the levels of IL- $1 \beta$ at $2 \mathrm{~h}$ and HMGB1 at $24 \mathrm{~h}$ in supernatants were assayed using ELISA ( $n=3,{ }^{*}, p<0.05$ versus LPS group). (C-D) Plumbagin (3 $\mu$ mol/L) inhibited LPS (100 ng/mL)-induced PKM2 mRNA expression and indicated cytokine release in NOX4-overexpressed BMDMs $\left(n=3,{ }^{*}, p<0.05\right)$.

treatment following CLP. The serum level of early cytokines such as IL-1 $\beta$ did not change at the late stage of CLP (Figure 6F). In addition, ECAR showed that the PMs isolated from the plumbagin treatment group had lower levels of glycolysis (Figure 6G).

Next, we determined whether pretreatment with plumbagin prevents CLP-mediated sepsis. Repetitive administration of plumbagin at -2 and $+6 \mathrm{~h}$ following the onset of sepsis conferred significant protection against CLP-induced lethal sepsis (Supplementary Figure S1A) with decreased serum levels of lactate (Supplementary Figure S1B), IL-1 $\beta$ (Supplementary Figure S1C), TNF
(Supplementary Figure S1D) and HMGB1 (Supplementary Figure S1E) at early and late stages. Moreover, the mRNA expression of PKM2 (Supplementary Figure S1F), NOX activity (Supplementary Figure S1G) and ECAR (Supplementary Figure S1H) in PMs isolated from CLP mice were lower after administration of plumbagin. 
A

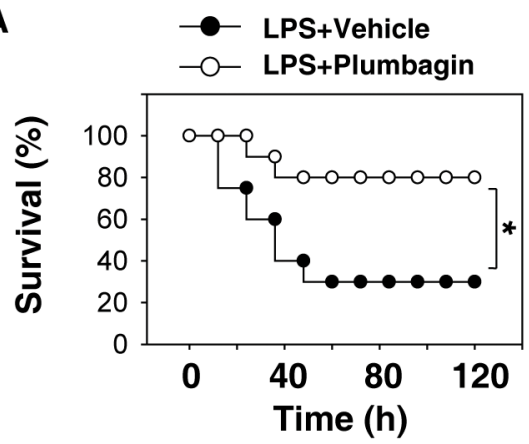

D

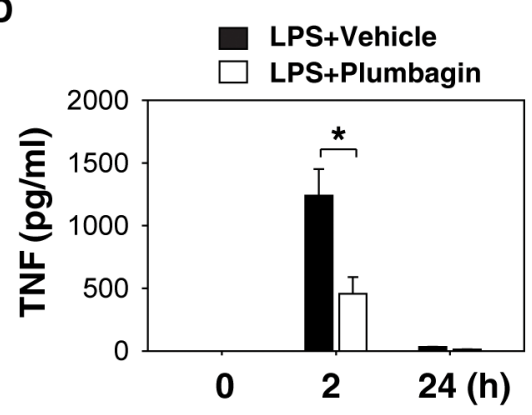

G

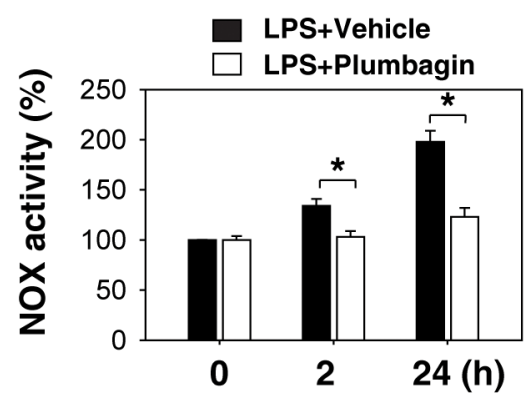

B

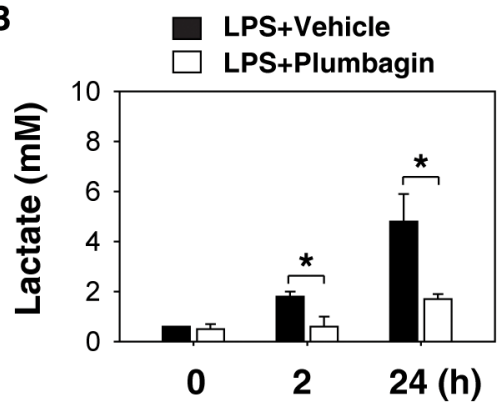

E

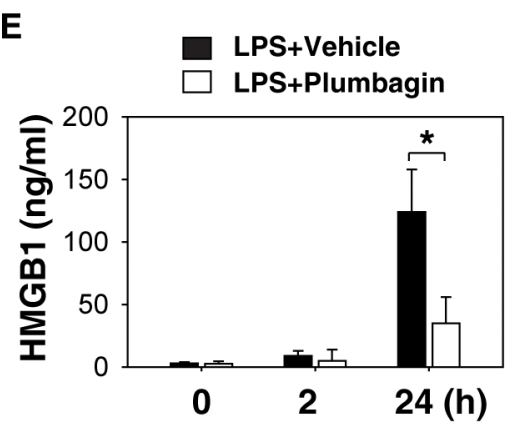

H

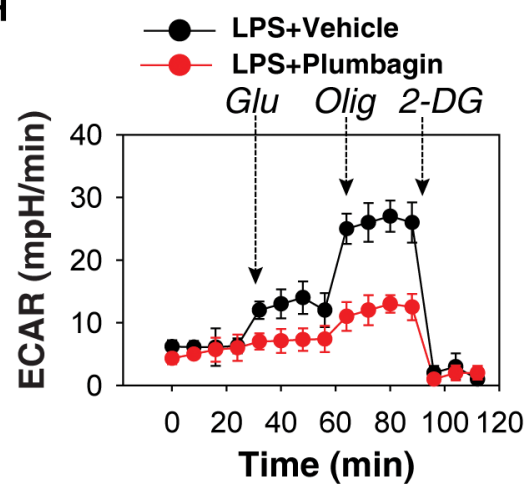

C

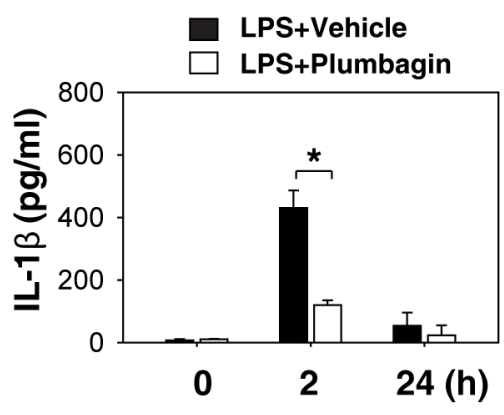

$\mathbf{F}$

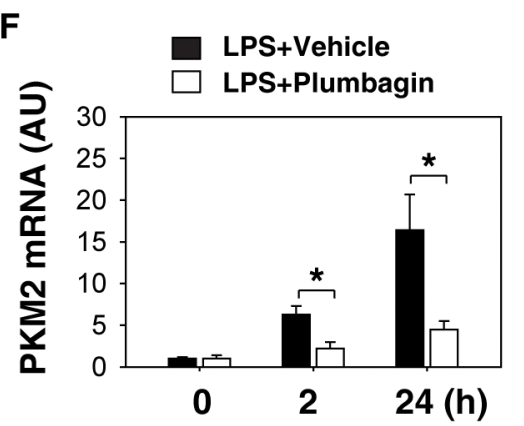

Figure 5. Plumbagin protects mice from endotoxic shock. (A) Mice $(n=20 /$ group) were injected with a single dose of plumbagin ( $4 \mathrm{mg} / \mathrm{kg}$ ), followed $30 \mathrm{~min}$ later by an infusion of endotoxin (lipopolysaccharide (LPS), $5 \mathrm{mg} / \mathrm{kg}$, intraperitoneally), and were then re-treated with plumbagin 12 and $24 \mathrm{~h}$ later. The Kaplan-Meier method was used to compare the differences in survival rates between groups (*, p < 0.05). (B-H) In parallel experiments, serum levels of lactate (B), IL-1B (C), TNF (D), HMGB1 (E), PKM2 mRNA (F), NOX activity $(G)$ and ECAR $(H)$ in isolated peritoneal macrophages at indicated time points were measured $(n=3-5$ animals/group, values are mean \pm SEM, ${ }^{*}, p<0.05$ ).

Collectively, these findings suggest that plumbagin protects against experimental lethal endotoxic shock and sepsis partly by inhibiting glycolysis-mediated proinflammatory cytokine release.

\section{DISCUSSION}

Sepsis is defined as infection with a systemic proinflammatory response and remains a major cause of mortality worldwide (36). In this study, we demonstrated that plumbagin significantly inhibits the systemic release of both early (IL-1 $\beta$ ) and late (HMGB1) cytokines, which are known to mediate the lethality of sepsis. The plumbagin-mediated protection is partly attributable to its inhibition of NOX4-mediated PKM2 expression and aerobic glycolysis. These findings provide a novel mechanism by which plumbagin exerts its antiinflammatory actions partly by modulating an immunometabolism in innate immune cells.

Aerobic glycolysis (also termed the Warburg effect) enables the conversion of glucose to lactate for ATP generation, even in the presence of normal levels of oxygen (37). This process was first observed in tumor cells to meet their increased energy needs for rapid proliferation. Like cancer cells, immune cells, including macrophages, dendritic cells 
A

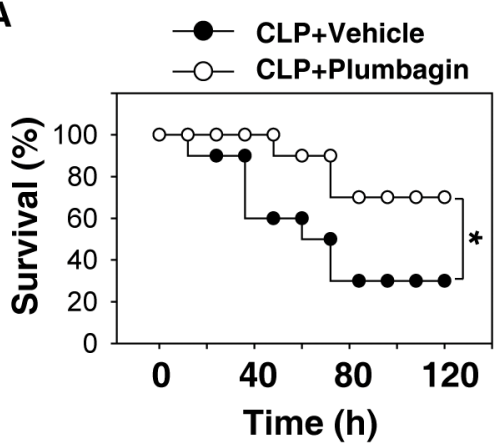

D

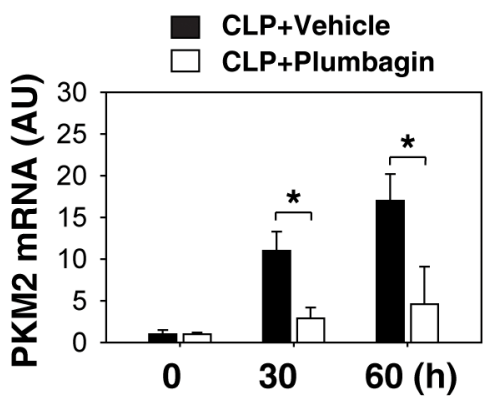

G

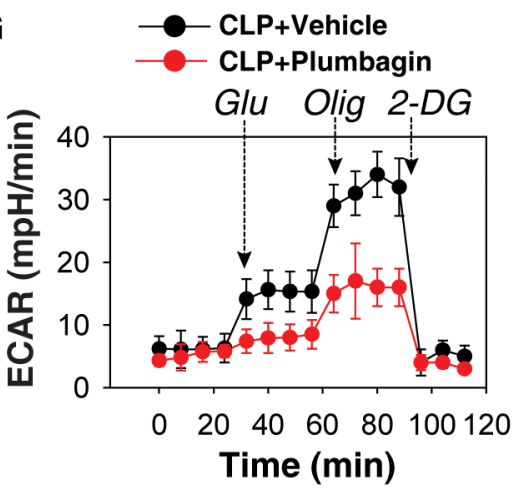

B

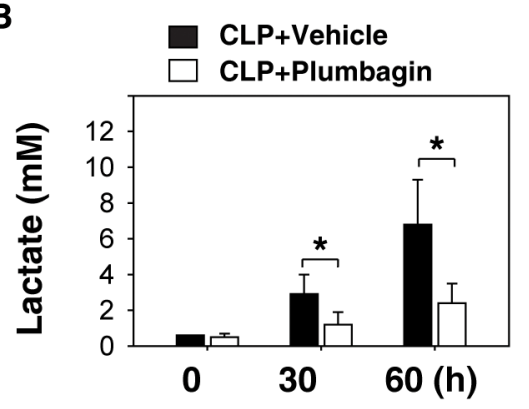

E

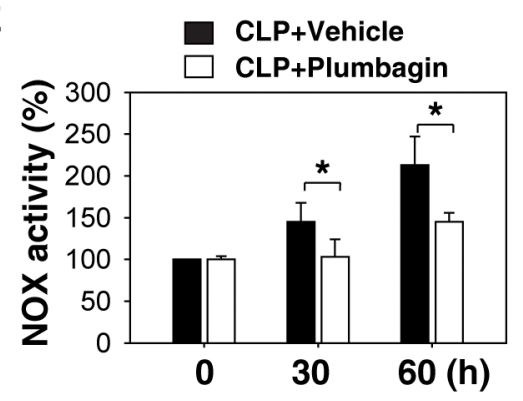

C

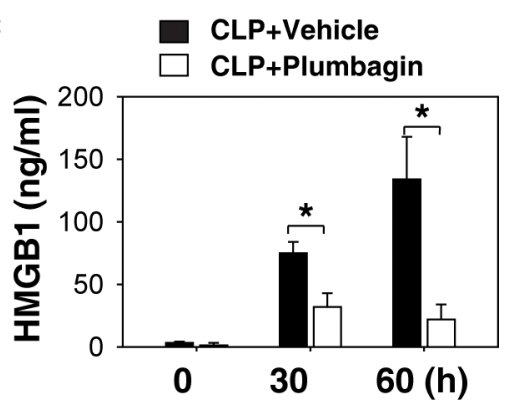

$\mathbf{F}$

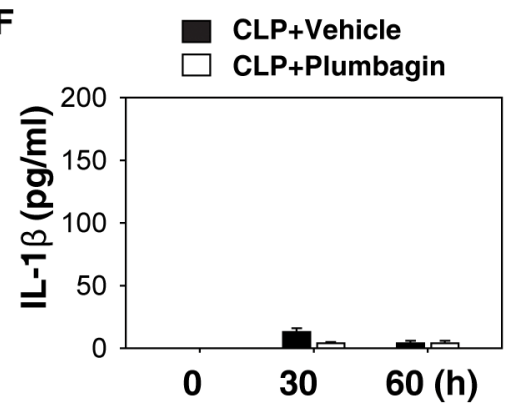

Figure 6. Delayed treatment with plumbagin protects mice from sepsis. (A) The cecal ligation and puncture (CLP) technique was used to induce intraabdominal sepsis in mice ( $n=20 /$ group). Repeated administration of plumbagin $(4 \mathrm{mg} / \mathrm{kg})$ at 24,48 , and $72 \mathrm{~h}$ after CLP significantly increased survival compared with vehicle group ( $\left.{ }^{*}, p<0.05\right)$, as measured by Kaplan-Meier test. (B-G) In parallel experiments, serum levels of lactate (B), IL-1 $\beta$ (F), HMGB1 (C) and PKM2 mRNA (D), NOX activity (E) and ECAR (G) in isolated peritoneal macrophages at indicated time points were measured $(n=3-5$ animals/group, values are mean \pm SEM, *,$p<0.05)$.

and T cells, also switch their energy production pathway from oxidative phosphorylation to aerobic glycolysis under inflammatory conditions (38-40). Inhibition of aerobic glycolysis limits LPS-induced macrophage activation and proinflammatory cytokine (for example, IL-1 $\beta$ and HMGB1) release $(10,26,27)$. These data suggest that immune cell activation in response to infection is a metabolic reprogramming event.
Targeting aerobic glycolysis might be a new strategy for the treatment of inflammatory diseases.

Plumbagin has been safely used for centuries in Indian and Chinese medicine as an anti-microbial, antiinflammatory and anticancer agent (41). Previous studies indicate that the antiinflammatory effects of plumbagin attributable to the inhibition of mitogen-activated protein kinases and nuclear factor (NF)- $\kappa \mathrm{B}$
(13-15) or activation of nuclear factor (erythroid-derived 2)-like 2 (42). Our current studies suggest that plumbagin can also suppress PKM2 expression in a NOX4-dependent manner. It has been recently shown that increased PKM2 expression promotes the metabolism of glucose by aerobic glycolysis and contributes to anabolic metabolism in macrophages $(10,26)$. Here, we demonstrated that plumbagin inhibited 
aerobic glycolysis and the release of IL-1 $\beta$ and HMGB1 in vitro and in vivo. Interestingly, PKM2 regulates IL-1 $\beta$ and HMGB1 release in a different manner. Monomeric or dimeric PKM2 can translocate to the nucleus, where it will interact with HIF1 $\alpha$ to promote IL-1 $\beta$ transcription (26). In contrast, PKM2 promotes HMGB1 release in a transcription-independent manner (10). The elevated production of lactate from PKM2-mediated glycolysis inhibits histone deacetylase activity and promotes HMGB1 acetylation, a critical event triggering HMGB1 translocation from the nucleus to the cytosol, and is then released to the extracellular space (10).

NOX4 is a constitutive NADPH oxidase that binds to $\mathrm{p} 22$ phox for generating superoxide in many cells including endothelial cells, fibroblasts, monocytes and macrophages. The activation of NOX4 is needed to modulate the redox signaling pathways involved in cell death, metabolism, and inflammation. For example, NOX4 is activated following cellular energy stress, and NOX4-dependent ROS production promotes autophagy in cardiomyocytes (43). Our study indicates that the activation of NOX4 is required for LPS-induced PKM2 expression in macrophages. Like knockdown of NOX4 by shRNA, pharmacological inhibition of NOX4 activity by plumbagin and GKT137831 also attenuates LPS-induced PKM2 expression, aerobic glycolysis, and proinflammatory cytokine (IL-1 $\beta$ and HMGB1) release. Our current study cannot exclude the possibility that plumbagin attenuates IL-1 $\beta$ secretion partly by inhibiting NF- $\kappa \mathrm{B}$ activation, which is required for increased PKM2 expression in epidermal growth factor-induced glycolysis in cancer cells (44). Furthermore, NOX4 is required for activation of NF- $\kappa \mathrm{B}$ in response to hypoxia and LPS $(45,46)$. It is possible that the NOX4-mediated PKM2 expression may depend on the activation of NF- $\kappa \mathrm{B}$ in LPS-induced macrophages.

\section{CONCLUSION}

In summary, we provide compelling evidence to support a novel mechanism and therapeutic potential for plumbagin in regulating PKM2-dependent glycolysis and protection of mice against lethal endotoxemia and sepsis. Plumbagin protected mice from lethal endotoxemia and polymicrobial sepsis partly through inhibiting glycolysis and proinflammatory cytokine release. Results from this study also show a novel role of NOX4 in immunometabolism by modulating PKM2 expression. Thus, pharmacological targeting of the NOX4/PKM2 pathway may be an attractive therapeutic strategy for the clinical management of sepsis and other inflammatory conditions.

\section{ACKNOWLEDGMENTS}

We thank Christine Heiner (Department of Surgery, University of Pittsburgh) for her critical reading of the manuscript. This work was supported by the National Institutes of Health of USA (R01GM115366 and R01CA160417 to DT; R01AT005076 and R01GM063075 to HW; and R01GM44100 to TB), grants from The National Natural Sciences Foundation of China (30973234 and 31171328 to LC), a grant from the Medical Scientific Research Foundation of the Guangdong Province of China (A2013585 to ZZ), and the National Natural Science Foundation of Guangdong (2016A030308 to DT).

\section{DISCLOSURE}

The authors declare that they have no competing interests as defined by Molecular Medicine, or other interests that might be perceived to influence the results and discussion reported in this paper.

\section{REFERENCES}

1. Riedemann NC, Guo RF, Ward PA. (2003) The enigma of sepsis. J. Clin. Invest. 112:460-467.

2. Dinarello CA, Thompson RC. (1991) Blocking IL-1: interleukin 1 receptor antagonist in vivo and in vitro. Immunol. Today. 12:404-410.

3. Wang H, et al. (1999) HMG-1 as a late mediator of endotoxin lethality in mice. Science. 285:248-251.

4. Bianchi ME. (2009) HMGB1 loves company. J. Leukoc. Biol. 86:573-576.
5. Andersson U, Tracey KJ. (2011) HMGB1 is a therapeutic target for sterile inflammation and infection. Annu. Rev. Immunol. 29:139-162.

6. Kang R, et al. (2014) HMGB1 in health and disease. Mol. Aspects Med. 40:1-116.

7. O'Neill LA, Hardie DG. (2013) Metabolism of inflammation limited by AMPK and pseudo-starvation. Nature. 493:346-355.

8. Wang R, Green DR. (2012) Metabolic checkpoints in activated T cells. Nat. Immunol. 13:907-915.

9. Biswas SK, Mantovani A. (2012) Orchestration of metabolism by macrophages. Cell Metab. 15:432-437.

10. Yang L, et al. (2014) PKM2 regulates the Warburg effect and promotes HMGB1 release in sepsis. Nat. Commun. 5:4436.

11. Checker R, Gambhir L, Sharma D, Kumar M, Sandur SK. (2015) Plumbagin induces apoptosis in lymphoma cells via oxidative stress mediated glutathionylation and inhibition of mitogen-activated protein kinase phosphatases (MKP1/2). Cancer Lett. 357:265-278.

12. Hsu YL, Cho CY, Kuo PL, Huang YT, Lin CC. (2006) Plumbagin (5-hydroxy-2-methyl-1,4naphthoquinone) induces apoptosis and cell cycle arrest in A549 cells through p53 accumulation via c-Jun NH2-terminal kinase-mediated phosphorylation at serine 15 in vitro and in vivo. J. Pharmacol. Exp. Ther. 318:484-494.

13. Wang T, et al. (2014) Plumbagin inhibits LPSinduced inflammation through the inactivation of the nuclear factor-kappa B and mitogen activated protein kinase signaling pathways in RAW 264.7 cells. Food Chem. Toxicol. 64:177-183.

14. Checker R, et al. (2014) Plumbagin, a vitamin K3 analogue, abrogates lipopolysaccharide-induced oxidative stress, inflammation and endotoxic shock via NF-кB suppression. Inflammation. 37:542-554.

15. Checker R, et al. (2010) Plumbagin inhibits proliferative and inflammatory responses of $\mathrm{T}$ cells independent of ROS generation but by modulating intracellular thiols. J. Cell. Biochem. 110:1082-1093.

16. Andujar I, Rios JL, Giner RM, Miguel Cerda J, Recio Mdel C. (2012) Beneficial effect of shikonin on experimental colitis induced by dextran sulfate sodium in BALB/c mice. Evid. Based Complement Alternat. Med. 2012:271606.

17. Weischenfeldt J, Porse B. (2008) Bone marrow-derived macrophages (BMM): isolation and applications. CSH Protoc. 2008:pdb prot5080.

18. Wu M, et al. (2007) Multiparameter metabolic analysis reveals a close link between attenuated mitochondrial bioenergetic function and enhanced glycolysis dependency in human tumor cells. Am. J. Physiol. Cell Physiol. 292:C125-C136.

19. Tang D, et al. (2011) High-mobility group box 1 is essential for mitochondrial quality control. Cell Metab. 13:701-711.

20. Tang D, et al. (2007) The anti-inflammatory effects of heat shock protein 72 involve inhibition of high-mobility-group box 1 release and proinflammatory function in macrophages. J. Immunol. 179:1236-1244. 
21. Wang X, et al. (2011) NADPH oxidase activation is required in reactive oxygen species generation and cell transformation induced by hexavalent chromium. Toxicol. Sci. 123:399-410.

22. Ulloa L, et al. (2002) Ethyl pyruvate prevents lethality in mice with established lethal sepsis and systemic inflammation. Proc. Natl. Acad. Sci. U. S. A. 99:12351-12356

23. Rittirsch D, Huber-Lang MS, Flierl MA, Ward PA. (2009) Immunodesign of experimental sepsis by cecal ligation and puncture. Nat. Protoc. 4:31-36.

24. Toscano MG, Ganea D, Gamero AM. (2011) Cecal ligation puncture procedure. J. Vis. Exp. 51:pii.

25. Zhang D, et al. (2014) 2-Deoxy-D-glucose targeting of glucose metabolism in cancer cells as a potential therapy. Cancer Lett. 355:176-183.

26. Palsson-McDermott EM, et al. (2015) Pyruvate kinase M2 regulates Hif- $1 \alpha$ activity and IL-1 $\beta$ induction and is a critical determinant of the warburg effect in LPS-activated macrophages. Cell Metab. 21:65-80.

27. Tannahill GM, et al. (2013) Succinate is an inflammatory signal that induces IL- $1 \beta$ through HIF-1 $\alpha$. Nature. 496:238-242.

28. Pendyala S, Natarajan V. (2010) Redox regulation of Nox proteins. Respir. Physiol. Neurobiol. 174:265-271.

29. Lee CF, Qiao M, Schroder K, Zhao Q, Asmis R. (2010) Nox4 is a novel inducible source of reactive oxygen species in monocytes and macrophages and mediates oxidized low density lipoprotein-induced macrophage death. Circ. Res. 106:1489-1497.

30. Guida M, et al. (2013) Inhibition of nuclear Nox4 activity by plumbagin: effect on proliferative capacity in human amniotic stem cells. Oxid. Med. Cell Longev. 2013:680816.

31. Yong R, et al. (2013) Plumbagin ameliorates diabetic nephropathy via interruption of pathways that include NOX4 signalling. PLOS ONE. 8:e73428.

32. Ding $Y$, et al. (2005) Inhibition of Nox-4 activity by plumbagin, a plant-derived bioactive naphthoquinone. J. Pharm. Pharmacol. 57:111-116.

33. Deliyanti D, Wilkinson-Berka JL. (2015) Inhibition of NOX1/4 with GKT137831: a potential novel treatment to attenuate neuroglial cell inflammation in the retina. J. Neuroinflammation. 12:136.

34. Green DE, et al. (2012) The Nox4 inhibitor GKT137831 attenuates hypoxia-induced pulmonary vascular cell proliferation. Am. J. Respir. Cell Mol. Biol. 47:718-726.

35. Melo AM, et al. (1974) [First observations on the topical use of Primin, Plumbagin and Maytenin in patients with skin cancer]. Revista do Instituto de Antibioticos, Universidade Federal de Pernambuco. 14:9-16.

36. Cohen J, et al. (2015) Sepsis: a roadmap for future research. Lancet Infect. Dis. 15:581-614.

37. Vander Heiden MG, Cantley LC, Thompson CB. (2009) Understanding the Warburg effect: the metabolic requirements of cell proliferation. Science. 324:1029-1033.
38. Krawczyk CM, et al. (2010) Toll-like receptorinduced changes in glycolytic metabolism regulate dendritic cell activation. Blood. 115:4742-4749.

39. Shi LZ, et al. (2011) HIF1alpha-dependent glycolytic pathway orchestrates a metabolic checkpoint for the differentiation of TH17 and Treg cells. J. Exp. Med. 208:1367-1376.

40. Dang EV, et al. (2011) Control of T(H)17/T(reg) balance by hypoxia-inducible factor 1 . Cell. 146:772-784.

41. Padhye S, Dandawate P, Yusufi M, Ahmad A, Sarkar FH. (2012) Perspectives on medicinal properties of plumbagin and its analogs. Med. Res. Rev. 32:1131-1158.

42. Son TG, et al. (2010) Plumbagin, a novel Nrf2/ ARE activator, protects against cerebral ischemia. J. Neurochem. 112:1316-1326.

43. Sciarretta S, et al. (2013) Activation of NADPH oxidase 4 in the endoplasmic reticulum promotes cardiomyocyte autophagy and survival during energy stress through the protein kinase RNA-activated-like endoplasmic reticulum kinase/eukaryotic initiation factor 2alpha/ activating transcription factor 4 pathway. Circ. Res. 113:1253-1264.

44. Yang W, et al. (2012) EGFR-induced and PKC $\varepsilon$ monoubiquitylation-dependent NF- $\kappa \mathrm{B}$ activation upregulates PKM2 expression and promotes tumorigenesis. Mol. Cell. 48:771-784.

45. Zhao QD, et al. (2015) NADPH oxidase 4 induces cardiac fibrosis and hypertrophy through activating Akt/mTOR and NFKB signaling pathways. Circulation. 131:643-655.

46. Lu X, et al. (2013) Hypoxia downregulates PPAR $\gamma$ via an ERK1/2-NF-кB-Nox4-dependent mechanism in human pulmonary artery smooth muscle cells. Free Radic. Biol. Med. 63:151-160.

Cite this article as: Zhang Z, et al. (2016) Plumbagin protects mice from lethal sepsis by modulating immunometabolism upstream of PKM2. Mol. Med. 22:162-72. 